\title{
Efeitos da cinesioterapia sobre os níveis de IGF-1, força muscular e autonomia funcional em mulheres idosas
}

\author{
Effects of kinesiotherapy on the levels of IGF-1, muscle strength \\ and functional autonomy in older women
}

\author{
Lidianne Teixeira Aires Santos \\ Rodrigo Gomes de Souza Vale \\ Danielli Braga de Mello \\ Tânia Santos Giani \\ Estélio Henrique Martin Dantas
}

1. Universidade Castelo Branco. Laboratório de Biociências da Motricidade Humana. Rio de Janeiro, RJ. Brasil.

2. Escola de Educação Física do Exército. Rio de Janeiro, RJ. Brasil.

3. Universidade Estácio de Sá. Rede Euro Americana de Motricidade Humana. Rio de Janeiro, RJ. Brasil.

Recebido em 05/06/09 Revisado em 28/11/09 Aprovado em 09/02/10
Resumo - Os níveis de fator de crescimento insulina símile tipo 1 (IGF-1), força muscular e autonomia funcional, tendem a declinar com a idade. O presente estudo teve como objetivo avaliar os efeitos da cinesioterapia sobre os níveis de IGF-1, força muscular e autonomia funcional em mulheres idosas com disfunções musculoesqueléticas. A amostra de 52 idosas foi dividida aleatoriamente em dois grupos iguais: Grupo Experimental (GE, idade: 68,52 \pm 4,68 anos; IMC: 28,05 $\pm 20,83$ ), submetido a 12 semanas de cinesioterapia, e Grupo Controle (GC, idade: 67,52 \pm 7,34 anos; IMC: 26,96 \pm 4,01). Foram avaliados os níveis séricos de IGF-1 através do método de Quimioluminescência e de força muscular através do teste de uma repetição máxima (1RM) nos movimentos de flexão (FQ) e extensão de quadril (EQ), flexão (FO) e extensão de ombro (EO) e a autonomia funcional, através do protocolo GDLAM. A ANOVA de medidas repetidas mostrou que o GE aumentou os níveis de IGF-1 ( $\triangle=19,74$ $\mathrm{ng} / \mathrm{ml} ; \mathrm{p}=0,028)$ do pré para o pós-teste. $\mathrm{O}$ GE aumentou os níveis de força muscular nos exercícios de flexão $(\triangle \mathrm{FO}=1,26 \mathrm{~kg} ; \mathrm{p}=0,001)$ e extensão de ombros $(\triangle \mathrm{EO}=1,90 \mathrm{~kg} ; \mathrm{p}=0,0001)$ e de extensão $(\triangle E Q=2,52 \mathrm{~kg} ; \mathrm{p}=0,0001)$ e flexão de quadril $(\triangle \mathrm{FQ}=2,82 \mathrm{~kg} ; \mathrm{p}=0,0001)$ do pré para o pós-teste. O GE reduziu os escores do IG $(\Delta=-16,73$; p =0,004) do pré para o pós-teste. Concluiu-se que, através do exercício resistido, podem-se obter resultados positivos sobre os níveis de IGF-1, força muscular e autonomia funcional em mulheres idosas com disfunção musculo-esquelética.

Palavras-chave: Autonomia funcional; Idosas; IGF-1; Força muscular.

Abstract - The levels of insulin-like growth factor-1 (IGF-1), muscle strength and functional autonomy tend to decrease with age. The objective of this study was to evaluate the effects of kinesiotherapy on IGF-1 levels, muscle strength and functional autonomy in older women with musculoskeletal dysfunction. A sample of 52 women was randomly divided into two groups: an experimental group (age: $68.52 \pm 4.68$ years, BMI: $28.05 \pm 20.83$ ) submitted to 12 weeks of exercise, and a control group (age: $67.52 \pm 7.34$ years; BMI: $26.96 \pm 4.01$ ). Serum IGF-1 levels were measured by a chemiluminescence method. Muscle strength was evaluated by one-repetition maximum (1-RM) testing of hip flexion (HF) and extension (HE) and shoulder flexion (SF) and extension (SE). The GDLAM protocol was used for the evaluation of functional autonomy. Repeated measures ANOVA showed an increase in IGF-1 levels after kinesiotherapy in the experimental group $(\Delta=19.74 \mathrm{ng} / \mathrm{ml}, \mathrm{p}=0.028)$. In the experimental group, muscle strength for shoulder flexion $(\triangle \mathrm{SF}=1.26 \mathrm{~kg}, p=0.001)$ and extension exercise $(\triangle \mathrm{SE}=1.90 \mathrm{~kg}, p=$ $0.0001)$ and for hip extension $(\triangle \mathrm{HE}=2.52 \mathrm{~kg}, p=0.0001)$ and flexion $(\triangle \mathrm{HF}=2.82 \mathrm{~kg}, p=$ $0.0001)$ exercise increased after therapy. IG scores were reduced in the experimental group after therapy $(\Delta=-16.73, p=0.004)$. In conclusion, resistance exercise can improve IGF-1 levels, muscle strength and functional autonomy in older women with musculoskeletal dysfunction.

Key words: Functional autonomy; Older women; IGF-1; Muscle strength. 


\section{INTRODUÇÃO}

O envelhecimento populacional tende a proporcionar o aumento da incidência de doenças crônico-degenerativas, que podem tornar as pessoas dependentes de outras até para a realização de Atividades da Vida Diária (AVD)1.

À medida que os anos passam, aumenta no indivíduo a massa adiposa ao mesmo tempo em que a sua massa muscular diminui. As mulheres são as mais afetadas e os seus declínios funcionais podem ser percebidos, de forma mais acentuada, no período da menopausa. Isto pode ocorrer devido às secreções hormonais reduzidas que, associadas à diminuição das AVD, podem contribuir para a diminuição da força muscular².

A fraqueza muscular e/ou encurtamento muscular e a diminuição da amplitude dos movimentos podem levar a alterações no equilíbrio e dores articulares, com consequente restrição da capacidade funcional e aumento do risco de queda e fraturas ${ }^{3}$.

As alterações musculares estão relacionadas aos níveis de produção do fator de crescimento insulina símile tipo 1 (IGF-1). O hormônio do crescimento $(\mathrm{GH})$ estimula a produção de IGF-14. Com o envelhecimento, há uma redução nesses níveis, principalmente, em mulheres ${ }^{5}$. A diminuição da liberação pulsátil do GH inibe a liberação do IGF-1. A nutrição desequilibrada, a presença de doenças crônicas, a ingestão de álcool, a disfunção do fígado e os baixos níveis de atividade física são fatores que podem comprometer a liberação de IGF-16.

Neste sentido, o exercício físico pode ser uma estratégia para estimular a secreção de IGF-1, principalmente, por apresentar, através do treinamento de força, uma alta correlação positiva com aumentos séricos do IGF-17. A força permite ao idoso realizar suas AVD com menos estresse fisiológico ${ }^{8}$. Desta forma, a participação dos idosos em atividades físicas regulares pode minimizar os declínios funcionais com o avançar da idade ${ }^{9}$. No entanto, para idosos com disfunção musculoesquelética, a cinesioterapia pode atuar no aumento da força muscular do idoso, por se tratar da realização de movimentos físicos planejados, e ajudar na prevenção e melhora de doenças crônico- degenerativas ${ }^{10}$.

Sendo assim, o objetivo do presente estudo foi avaliar os efeitos da cinesioterapia sobre os níveis de IGF-1, força muscular e autonomia funcional em mulheres idosas com disfunções musculoesqueléticas.

\section{PROCEDIMENTOS METODOLÓGICOS}

\section{Amostra}

A pesquisa foi do tipo experimental em que o universo das 123 mulheres idosas, residentes no Abrigo São Lucas, situado no bairro Cacimba Velha, em Teresina (PI), foram convidadas a participar do presente estudo. Foram utilizados como critérios de inclusão: presença de disfunção musculo-esquelética (osteoporose, artrose, tendinite, entre outras); capacidade de realizar as AVD sem ajuda de terceiros; não praticar atividade física no período mínimo de 12 meses; e apresentar níveis de IGF-1 normal para a idade ${ }^{11}$.

Foram excluídas as idosas consideradas inaptas à realização dos testes de força e de autonomia funcional, depois de passarem pela avaliação médica.

Após a aplicação dos critérios de inclusão e exclusão, 52 mulheres idosas, com idade compreendida entre 60 e 86 anos (idade: 68,92 $\pm 6,0$ anos; IMC: $26,04 \pm 1,45)$ compuseram a amostra. Os sujeitos foram divididos em dois grupos - Experimental e Controle-, e escolhidos aleatoriamente (Tabela 1).

As idosas participantes do estudo assinaram o Termo de Consentimento Livre e Esclarecido de acordo com a Resolução 196/96 do Conselho Nacional de Saúde e a declaração de Helsinki. ${ }^{12}$ O estudo foi aprovado pelo Comitê de Ética da Pesquisa da Universidade Castelo Branco sob n ${ }^{\circ}$ 0165/2008.

Tabela 1: Características da amostra.

\begin{tabular}{llccccc}
\hline & & Média & DP & Mínimo & Máximo & valor-p (SW) \\
\hline \multirow{3}{*}{ GE $(\mathrm{n}=26)$} & Idade & 68,52 & 4,68 & 61,00 & 75,00 & 0,052 \\
& Massa Corporal & 63,99 & 46,00 & 46,00 & 92,60 & 0,105 \\
& Estatura & 1,51 & 1,40 & 1,40 & 1,64 & 0,056 \\
\multirow{3}{*}{ GC $(\mathrm{n}=26)$} & IMC & 28,05 & 20,83 & 20,83 & 43,44 & 0,068 \\
& Idade & 67,52 & 7,34 & 60,00 & 86,00 & 0,051 \\
& Massa Corporal & 67,45 & 7,07 & 57,00 & 87,00 & 0,055 \\
& Estatura & 1,59 & 0,05 & 1,47 & 1,65 & 0,051 \\
& IMC & 26,96 & 4,01 & 21,68 & 39,72 & 0,061 \\
\hline
\end{tabular}

DP: desvio-padrão; GE: grupo experimental; GC: grupo controle; IMC: Índice de massa corporal; SW: teste de Shapiro-Wilk. 
Procedimentos de coleta de dados

\section{- Avaliação Antropométrica}

Para avaliação da massa corporal, da estatura e cálculo do índice de massa corporal (IMC), foi utilizada uma balança mecânica com estadiômetro, precisão de 100 gramas e capacidade para 150 quilos, marca Filizola ${ }^{\circledR}$ (Brasil), conforme protocolo da International Society for the Advancement of Kinanthropometry ${ }^{13}$.

\section{- Avaliação da Autonomia Funcional}

A avaliação da autonomia funcional foi realizada pela aplicação de uma bateria de testes com quesitos necessários para avaliar o desempenho nas AVD, seguindo o protocolo GDLAM de autonomia ${ }^{14,15}$ a seguir: caminhar 10 metros $(\mathrm{C} 10 \mathrm{M})^{16}$; sentar-se e levantar-se da cadeira e locomover-se pela casa (LCLC) ${ }^{17}$; levantar-se da posição sentada $(\mathrm{LPS})^{18}$; levantar-se da posição decúbito ventral (LPDV) ${ }^{19}$; e vestir e tirar uma camiseta (VTC) ${ }^{20}$.

Os sujeitos executaram duas tentativas em cada teste com intervalo de cinco minutos entre elas, tendo sido registrada a melhor tentativa. Após a mensuração do tempo de cada teste, em segundos, foi calculado o índice GDLAM de autonomia (IG), através da seguinte fórmula ${ }^{14}$.

$$
I G=\frac{[(C 10 m+L P S+L P D V+D T C) \times 2]+L C L C}{4}
$$

Onde:

C10m, LPS, LPDV, VTC e LCLC $=$ tempo aferido em segundos. $\mathrm{IG}=$ índice GDLAM em escores.

\section{- Coleta sanguínea}

Para avaliar os níveis séricos de IGF-1, foi realizada, em um laboratório de análises clínicas, uma coleta sanguínea, utilizando-se algodão, álcool, agulha e seringa descartáveis, e tubo estéril; e foi aplicado o método de Quimioluminescência - IMMULITE - DPC MED LAB (sistema fechado - a vácuo). Os componentes dos grupos fizeram um jejum de 12 horas, e a coleta feita pela manhã, às 7h. O valor considerado padrão de referência para a idade da amostra foi fixado entre 69 a $200 \mathrm{ng} / \mathrm{ml} .^{11}$

Teste de uma Repetição Máxima (1-RM)

Para determinar a força muscular máxima, foi realizado o teste de uma repetição máxima - 1-RM $(r=0,92)^{21}$. Cada indivíduo foi orientado a realizar uma série de movimentos com o gesto motor que seria utilizado no teste (flexão e extensão de quadril, flexão e extensão de ombro). Houve três minutos de intervalo antes da primeira tentativa, sendo realizada uma repetição a cada tentativa. Entre cada tentativa executada, ou não, foi dado um intervalo de cinco minutos. Sempre que uma tentativa não fosse realizada, reduzia-se a carga. A avaliação da força foi aplicada pelo mesmo avaliador, cuja confiabilidade foi testada, de forma aleatória, com 10 sujeitos, uma semana antes do experimento, com intervalo de $72 \mathrm{~h}$ entre os testes, apresentando um coeficiente de correlação intraclasse (ICC) de 0,92.

Após o período de intervenção, os dois grupos (GC e GE) foram submetidos a todos os testes (pós-teste), seguindo os mesmos procedimentos e condições realizadas no início do estudo.

\section{- Intervenção}

Depois da avaliação inicial, as mulheres idosas do GE foram submetidas a 12 semanas de tratamento cinesioterapêutico, na forma de exercícios resistidos: flexão e extensão do ombro e flexão e extensão do quadril.

O programa foi do tipo alternado por segmento corporal, seguindo a ordem dos exercícios resistidos para os músculos flexores de ombro e de quadril, extensores de ombro e de quadril, todos realizados no mesmo dia. Os equipamentos usados foram halteres e caneleiras. A frequência foi de três vezes por semana, em dias alternados, com 50 minutos de duração por sessão. Foram utilizadas três séries de oito a 10 repetições, com intervalos de dois minutos entre as séries e entre os exercícios, e a carga entre 75 e $85 \%$ de 1-RM. A intensidade de esforço foi controlada através da utilização da escala de esforço percebido OMNI-RES ${ }^{22}$, mantendo-se a intensidade em um escore médio de 7,82 $\pm 2,52$ durante todo o período de intervenção. A velocidade de execução foi de lenta a moderada. Antes e após o treinamento, foram realizados exercícios de alongamentos.

As idosas do grupo controle foram orientadas a não praticarem nenhum tipo de atividade física regular durante o período do experimento. Logo após a obtenção dos resultados da pesquisa e o término do período experimental proposto, o grupo controle foi submetido à mesma intervenção que o grupo experimental.

\section{Tratamento Estatístico}

Os dados foram tratados pelos programas Excel e SPSS 14.0 for Windows e apresentados como média, desvio-padrão, e valores mínimos e máximos. Os testes de Shapiro-Wilk e Levene foram utilizados para a verificação da normalidade e homogeneidade de variância dos dados da amostra. Empregou-se a análise de variância de medidas repetidas nos 
fatores grupo (GE e GC) e tempo (pré e pós-teste), seguida do post hoc de Tukey para identificar as possíveis diferenças. $\mathrm{O}$ estudo admitiu o valor de $\mathrm{p}<0,05$ para a significância estatística.

\section{RESULTADOS}

A figura 1 apresenta os resultados das comparações intra e intergrupos do IGF-1. Observou-se que o GE aumentou os níveis de IGF-1 ( $\Delta=19,74$ $\mathrm{ng} / \mathrm{ml} ; \mathrm{p}=0,028)$ do pré para o pós-teste. $\mathrm{O} \mathrm{GE}$ também se mostrou com níveis mais elevados no pós-teste, quando comparado ao GC ( $\Delta=18,89 \mathrm{ng} /$ $\mathrm{ml} ; \mathrm{p}=0,045)$. O GC não obteve modificações nos níveis de IGF-1.

A figura 2 apresenta os resultados das comparações intra e intergrupos da força muscular, obtida através do teste de 1-RM. Observou-se que o GE aumentou os níveis de força muscular nos exercícios de flexão $(\triangle F O=1,26 \mathrm{~kg} ; \mathrm{p}=0,001)$ e extensão de ombros $(\triangle E O=1,90 \mathrm{~kg} ; \mathrm{p}=0,0001)$ e de extensão $(\triangle E Q=2,52 \mathrm{~kg} ; \mathrm{p}=0,0001)$ e flexão de quadril $(\triangle \mathrm{FQ}=2,82 \mathrm{~kg} ; \mathrm{p}=0,0001)$ do pré para o pós-teste. $\mathrm{O}$ GE também se mostrou com maiores níveis de força muscular no pós-teste, quando comparado ao GC $(\triangle \mathrm{FO}=1,70 \mathrm{~kg}, \mathrm{p}=0,0001 ; \Delta \mathrm{EO}=2,67 \mathrm{~kg}, \mathrm{p}=0,0001 ;$ $\Delta \mathrm{EQ}=3,04 \mathrm{~kg}, \mathrm{p}=0,0001 ; \Delta \mathrm{FQ}=2,72 \mathrm{~kg}, \mathrm{p}=0,0001)$. O GC não obteve modificações na força muscular.

A figura 3 apresenta os resultados das comparações intra e intergrupos da bateria de testes de autonomia funcional. Observou-se que o GE reduziu os tempos de execução nos testes $\mathrm{C} 10 \mathrm{~m}(\Delta=$ $-11,96 s ; \mathrm{p}=0,047), \operatorname{LPS}(\Delta=-4,88 \mathrm{~s} ; \mathrm{p}=0,008)$, LPDV $(\Delta=-2,52 \mathrm{~s} ; \mathrm{p}=0,002), \operatorname{VTC}(\Delta=-6,39 \mathrm{~s} ; \mathrm{p}=0,047) \mathrm{do}$ pré para o pós-teste. $\mathrm{O}$ GE também se mostrou com os melhores desempenhos nos testes de autonomia no pós-teste, quando comparado ao GC $(\triangle \mathrm{LPS}=$ $-6,68 \mathrm{~s}, \mathrm{p}=0,0001 ; \Delta \mathrm{LPDV}=-2,81 \mathrm{~s}, \mathrm{p}=0,001) . \mathrm{O}$ GC não obteve modificações no desempenho dos testes de autonomia.

A figura 4 apresenta os resultados das comparações intra e intergrupos do IG. Observou-se que o GE reduziu os escores do IG $(\Delta=-16,73 ; \mathrm{p}=0,004)$ do pré para o pós-teste. $\mathrm{O}$ GE também se apresentou com o melhor desempenho geral de autonomia no pós-teste, quando comparado ao $\mathrm{GC}(\triangle \mathrm{IG}=-13,30$; p=0,039). O GC não obteve modificações no IG.

\section{DISCUSSÃO}

De acordo com os resultados obtidos, a presente pesquisa mostrou redução nos tempos de execução dos testes de autonomia funcional e aumento nos

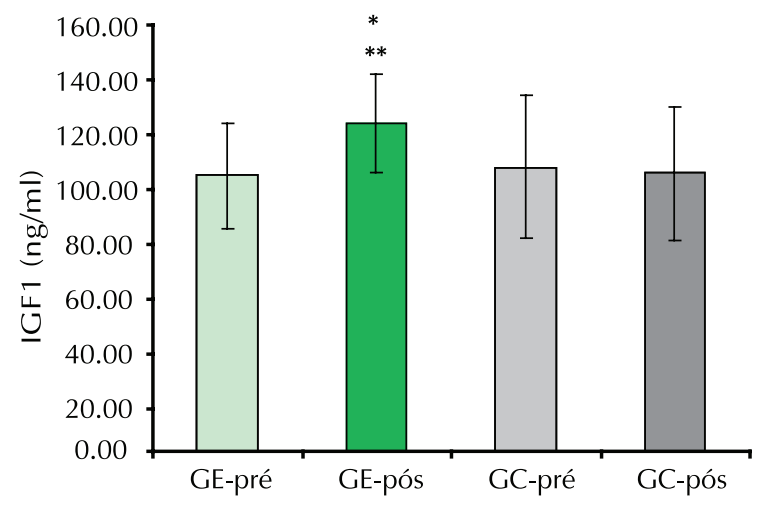

Figura 1: Análise das comparações do IGF-1 entre o grupo experimental (GE) e controle (GC). * p<0,05; GE-pré vs GE-pós; **p<0,05; GE-pós vs GC-pós.

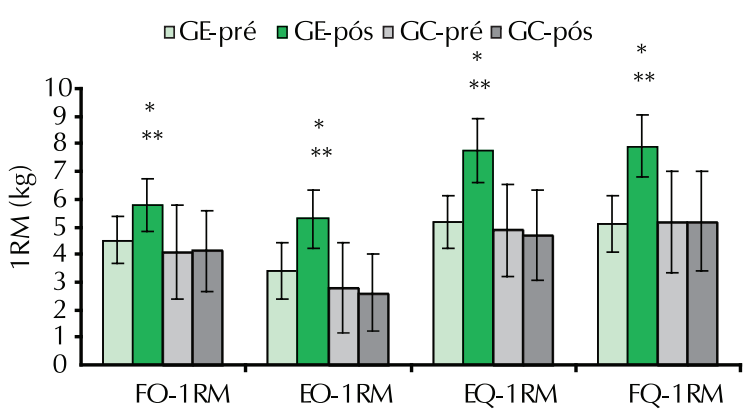

Figura 2: Análise das comparações da força muscular (1-RM) nos exercícios de flexão (FO) e extensão de ombros (EO) e extensão (EQ) e flexão do quadril (FQ) entre o grupo experimental (GE) e controle (GC). ${ }^{*} \mathrm{p}<0,05$; GE-pré vs GE-pós ; ${ }^{* *} \mathrm{p}<0,05$; GE-pós vs GC-pós.

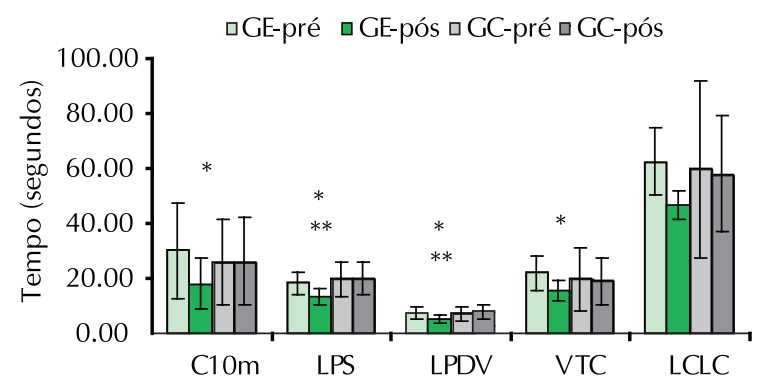

Figura 3: Análise das comparações dos testes de autonomia funcional entre os grupos experimental (GE) e controle (GC). $\mathrm{C} 10 \mathrm{~m}$ = caminhar 10 metros; LPS = levantar da posição sentada; LPDV = levantar da posição decúbito ventral; VTC = vestir e tirar a camisa; LCLC = levantar da cadeira e locomover-se pela casa; *p<0,05; GE-pré vs GE-pós ; **p<0,05; GE-pós vs GC-pós.

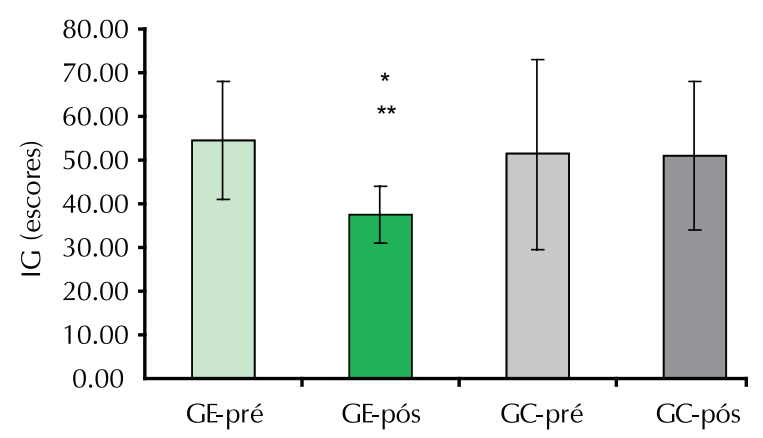

Figura 4: Análise das comparações do índice GDLAM de autonomia (IG) entre o grupo experimental (GE) e controle (GC). *p<0,05; GE-pré vs GE-pós; **p<0,05; GE-pós vs GC-pós. 
níveis de força muscular e IGF-1 no GE, após o período de intervenção com os exercícios resistidos. O GC não obteve alterações significativas nas variáveis analisadas. Isto pode ter ocorrido devido ao fato de as idosas serem institucionalizadas e não participarem de atividades físicas regulares, pois a diminuição da força com a idade está associada, principalmente, à redução da massa muscular e dos níveis de IGF-1 que, em consequência, podem reduzir a capacidade funcional dos idosos ${ }^{23}$.

Neste sentido, o treinamento de força tem se mostrado eficaz para minimizar os efeitos deletérios do envelhecimento sobre essas variáveis ${ }^{24}$. Dantas et al. ${ }^{25}$ observaram que mulheres idosas, que praticaram exercícios de alta intensidade na musculação, obtiveram maiores níveis de IGF-1, quando comparadas com as que praticaram meditação, corroborando, assim, os resultados do presente estudo.

A força muscular apresenta uma maior correlação com o IGF-126 e pode ser aumentada através de um programa de exercícios resistidos como ocorreu no presente estudo. Vale et al. ${ }^{23}$ conseguiram aumentos tanto na força muscular como nos níveis de IGF-1, realizando atividades com exercícios resistidos de alta intensidade, numa frequência de três vezes por semana, em mulheres idosas sedentárias, com idade média de 68 anos, quando comparadas com o grupo de exercícios aeróbicos formado por mulheres com as mesmas características. Esses achados comprovam os resultados obtidos no GE do presente estudo, em que os níveis de força muscular e de IGF-1 aumentaram após o tratamento cinesioterapêutico, não ocorrendo o mesmo com GC.

Silva et al. ${ }^{5}$ obtiveram aumento nos níveis de força muscular das idosas do grupo experimental, que realizaram treinamento de força por 12 semanas em alta intensidade (85\% 1-RM), e atribuíram esse ganho à fase miogênica da intervenção. $\mathrm{O}$ mesmo pode ter ocorrido no presente estudo, pois a intensidade utilizada pela escala OMNI-RES atendeu a um nível de esforço alto, justificando os achados encontrados no GE.

A atividade física tende a melhorar a aptidão funcional de idosos 9 . $O$ que foi corroborado pelo presente estudo com os resultados obtidos pelo GE nos testes de autonomia funcional que, após o tratamento cinesioterapêutico, realizado de forma resistida, obteve uma diminuição no tempo de execução dos testes. César et al. ${ }^{3}$ mostraram que indivíduos que praticam uma atividade física regular apresentam melhor condicionamento físico e melhor desempenho nas atividades que requerem força muscular, principalmente, de membros infe- riores, impactando diretamente na sua autonomia funcional nas atividades de vida diária.

A cinesioterapia através dos exercícios terapêuticos é considerada um elemento primordial na maioria dos planos de assistência da fisioterapia, por incluir, também, condicionamento aeróbico, treinamento de força, potência e endurance ${ }^{10}$.

Dantas e Vale ${ }^{15}$ demonstraram em seu estudo que, independente da atividade a ser realizada, a mesma pode promover uma melhora nos níveis de autonomia funcional da idosa. Entretanto, as atividades físicas de alta intensidade têm demonstrado melhores resultados quando comparadas com as de baixa ou moderada intensidade ${ }^{5,23,24}$. Os resultados encontrados no presente estudo comprovaram também que, após a intervenção de cinesioterapia, o GE obteve melhora no IG, que expressa de forma geral o nível de autonomia funcional, voltado para a realização das AVD de um indivíduo idoso, nos níveis de força muscular e IGF-1. Contudo, no que se refere às modificações hormonais causadas pelo exercício físico, existem outros fatores que podem influenciar os resultados aqui encontrados, mas que não foram investigados na presente pesquisa como o estresse, a ansiedade e o estado psicológico dos indivíduos ${ }^{27}$.

\section{CONCLUSÃO}

presente estudo mostrou que mulheres idosas com disfunção musculoesquelética, submetidas ao exercício resistido, apresentaram resultados positivos sobre os níveis de IGF-1, força muscular e autonomia funcional. Também foi possível observar que um programa cinesioterapêutico com aplicação de exercícios de alta intensidade proporcionou aumentos nos níveis séricos de IGF-1. Isto fortalece a importância da prática/realização de atividade física regular para o idoso, como forma de manutenção da autonomia funcional voltada para a execução de suas AVD sem ajuda de terceiros.

\section{REFERÊNCIAS BIBLIOGRÁFICAS}

1. Benedetti TRB, Mazo GZ, Gobbi S, Lopes MA, Gobbi LTB, Ferreira L, et al. Valores normativos de aptidão funcional em mulheres de 70 a 79 anos. Rev Bras Cineantropom Desempenho Hum 2007;9(1):28-36.

2. Gibney J, Healy M-L, Sönksen PH. The growth hormone/insulin-like growth factor-I axis in exercise and sport. Endocr Rev 2007;28(6):603-24.

3. César EP, Almeida OV, Pernambuco CS, Vale RGS, Dantas EHM. Aplicação de quatro testes do protocolo GDLAM - Grupo de Desenvolvimento Latino-Americano para Maturidade. R Min Educ Fís 2004;12(1):18-37. 
4. Moran S, Chen Y, Ruthie A, Nir Y. Alterations in IGF-1 effect elderly: role of physical activity. Eur Rev Aging Phys Act 2007;4(2):77-84.

5. Silva JGFB, Calvo XD, Soler EI, Dantas EHM. Efeitos do treinamento de força sobre os níveis de IGF-1 e de força muscular nas fases neurogênica e miogênica de idosas. Rev Bras Geriatr Gerontol 2009;12(1):35-48.

6. Rubin MR, Kraemer WJ, Maresh CM, Volek JS, Ratamess NA, Vanheest JL, et al. High-affinity growth hormone binding protein and acute heavy resistance exercise. Med Sci Sports Exerc 2005;37(3):395-03.

7. Oliveira LSC, Côrtes GG, Vale RGS, Dantas EHM. Níveis séricos de IGF-1 em gerontes. Fit Perf J 2003;2(5):289-92.

8. American College of Sports Medicine (ACSM). ACSM's guidelines for exercise testing and prescription. 7ed. Baltimore: ed. D. FACSM; 2006.

9. Rosa MF, Mazo GZ, Silva AH, Brust C. Efeito do período de interrupção de atividades aquáticas na aptidão funcional de idosas. Rev Bras Cineantropom Desempenho Hum 2008;10(3):237-42.

10. Hall CM, Brody LT. Exercício terapêutico: na busca da função. $2^{a}$ Ed. Rio de Janeiro: Guanabara Koogan; 2007.

11. Instituto de Patologia Clínica Hermes Pardini (IPCHP). Manual de Exames. Belo Horizonte; 2007.

12. World Medical Association. Declaration of Helsinki. Ethical Principles for Medical Research Involving Human Subjects. 59th WMA General Assembly, Seoul; October 2008.

13. Marfell-Jones M, Olds T, Stewart A, Carter L. International standards for anthropometric assessment. Potchefstroom, South África: ISAK; 2006.

14. Vale RGS, Dantas EHM. Autonomia funcional do idoso. In: Dantas EHM, Vale RGS. Atividade física e envelhecimento saudável. Rio de Janeiro: Shape,2008.

15. Dantas EHM, Vale RGS. Protocolo GDLAM de avaliação da autonomia. Fit Perf J 2004;3(3):169-80.

16. Sipilä S, Multanen J, Kallinen M, Era P, Suominen H. Effects of strength and endurance training on isometric muscle strength and walking speed in elderly women. Acta Physiol Scand 1996;156:457-64.

17. Andreotti RA, Okuma SS. Validação de uma bateria de testes de atividade da vida diária para idosos fisicamente independentes. Rev Paul Educ Fís 1999;1(13):46-66.

18. Guralnik JM, Simonsick EM, Ferrucci L, Glynn RJ, Berkman LF, Blazer DG, et al. A short physical performance battery assessing lower extremity function: association with self-reported disability and prediction of mortality and nursing home admission. J Gerontol 1994;49(2):85-94.
19. Alexander NB, Ulbric HJ, Raheja A, Channer D. Rising from the floors in older adults. J Am Geriatr Soc 1997;136(5):564-9.

20. Vale RGS, Pernambuco CS, Novaes JS, Dantas EHM. Teste de autonomia funcional: vestir e tirar uma camiseta (VTC). Rev Bras Cienc Mov 2006;14(3):71-8.

21. Goto KM, Nagasawa M, Yanagisawa O, Kisuka T, Ishii N, Takamatsu K. Muscular adaptations to combinations of high- and low-intensive resistance exercises. J Strength Cond Res 2004;18(4):730-7.

22. Robertson RJ, Goss FL, Rutkowski J, Lenz B, Dixon C, Timmer J, et al. Concurrent Validation of the OMNI Perceived Exertion Scale for Resistance Exercise. Med Sci Sports Exerc 2003;35(2):333-41.

23. Vale RGS, Oliveira RD, Pernambuco CS, Meneses YPSF, Novaes JS, Andrade AFD. Effects of muscle strength and aerobic training on basal serum levels of IGF-1 and cortisol in elderly women. Arch Gerontol Geriatr 2009;49:343-7.

24. Kostek M, Delmonico M. Muscle strength response to strength training is influenced by insulin-like growth factor 1 genotype in older adults. J Appl Physiol 2005;98(6):2147-54.

25. Dantas EH, Cruz TH, Castro JC, Bastos FA, Santos CA, Caetano LF. Serum levels of IGF-1 in elderly women engaged in various motor activities. Phys Educ Sport 2008;1(52):81-3.

26. Cordeiro LS, Fortes MS, Attie KF, Dantas EHM. Relação entre o nível sérico basal de GH e de IGF-1 e a autonomia e o estado de condicionamento físico da idosa ativa. Fit Perf J 2005;4(5):293-8.

27. Peer JM, Roelofs K, Spinhoven P. Cortisol administration enhances the coupling of midfrontal delta and beta oscillations. I J Psycho 2008;67:144-50. 